\title{
Contactless Excitation System for Synchronous Generators
}

\author{
Do-Hyun Kang ${ }^{1}$, Dae-Hyun Koo ${ }^{1}$, Ion Vadan ${ }^{2}$, Paul Curiac ${ }^{1}$ \\ ${ }^{1}$ Korea Electrotechnology Research Institute, KERI, PO BOX 20, Changwon, 641-600, KOREA, \\ Phone: +82-55-280-1405, FAX: +82-55-280-1547, \\ E-MAIL: dhkang@keri.re.kr \\ 2 Technical University of Cluj-Napoca, C. Daicoviciu str. 15, 3400 CLUJ-NAPOCA, ROMANIA, \\ Phone: +40-64-193161/130, FAX: +40-64-192055, \\ E-MAIL: Ioan.Vadan@eps.utcluj.ro
}

\begin{abstract}
The paper proposes a new application of contactless power supply technology to transfer electric power to rotating rotors of synchronous electric generators (excitation system, usually placed in rotor). The design and operation simulation of a magnetic collector, the main part of a contactless power supply of a synchronous machine excitation system is presented. This new excitation system, will keep the advantages of rapidity offered by static excitation combined with slip ring collector and the easy maintenance offered by brush-less excitation system, avoiding the maintenance problems of the first system and the high time constant of exciter coil of the later. An application for a synchronous generator of $500 \mathrm{~kW}$ is also presented.
\end{abstract}

\section{Key words}

Contactless Power Supply, Static Excitation System, Rotating Transformer, Synchronous Machines.

\section{Introduction}

In the last 10 years a new electric energy supplying technology, Contact-less Power Supply (CPS), became available [1-3] and thousands of this kind of devices now is working all round the world.

CPS supplies electrical current by means of electromagnetic induction, similar to the primary-to secondary energy conversion of a transformer. However, the primary and secondary windings of a conventional transformer are placed on a common ferromagnetic core. This geometry creates a high coupling coefficient but excludes any relative movement between the two windings. The CPS transformer, on the other hand, has the magnetic core split in two parts separated by an airgap; one carrying primary winding and one secondary winding and allows relative movement between the two. The reduced coupling of the open core geometry is compensated by a higher primary frequency. So the CPS electric circuit looks like in Fig. 1. The structure is a little complex, but the advantages of such a device are very important:

-Efficiency: Both primary and secondary circuits are resonant so that losses and harmonics are minimized and efficiencies of up to $96 \%$ are available.

- Power: Hundreds of kW-s may be transferred.

-Large Air-gap: Power may be transferred across airgaps of $100 \mathrm{~mm}$ and more.

-Maintenance: No brushes or rings wear ensures that the CPS system is virtually maintenance free.

-Data Transfer: Signal and data transfer is possible with CPS with minimal additional hardware. An integrated power and data system is currently being developed.

- Speed: with slip ring collector speed is a limiting factor. With CPS speed of operation is unlimited.

-Safety: All components are fully enclosed and insulated. Hence the system is fully touch proof.

- Sensitive Environments: The fact, that no carbon dust, or other wear or sparks is generated, make CPS suitable for sensitive or hazardous environments.

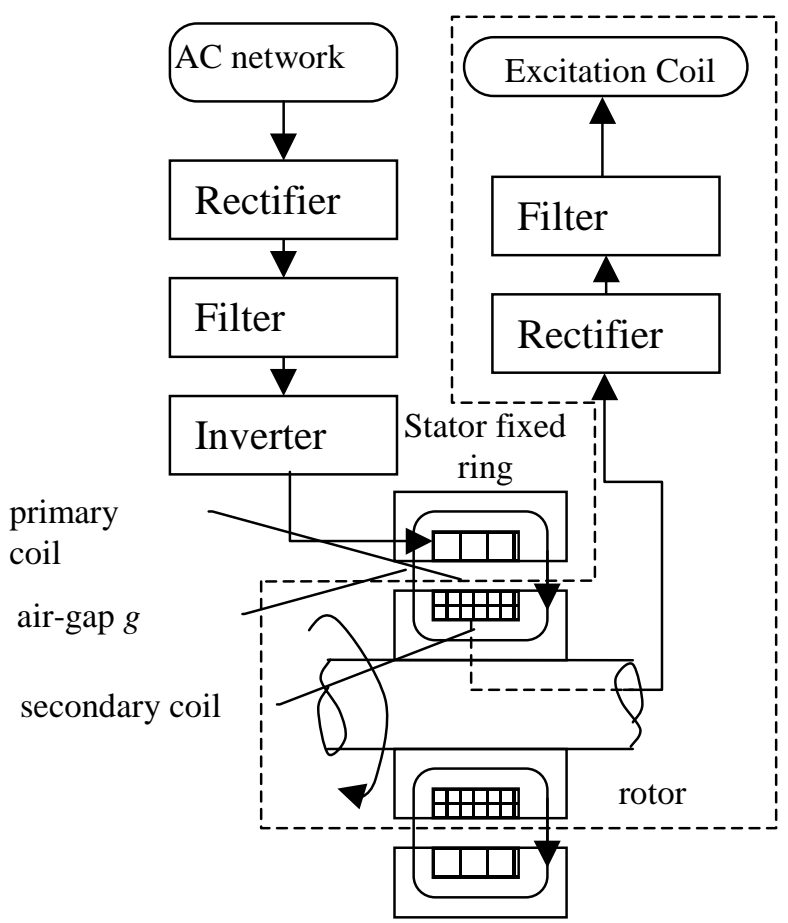

Fig. 1 CPS structure. 
The main part of CPS is a transformer, which can be of two types:

- Linear Inductive Power Transformer (LIPT), which transfers energy to a straight-line moving device [2-3];

-Rotating Transformer (RT), which transfers energy to a rotating device (Fig. 1).

\section{Synchronous System \\ Machine's Excitation}

A.C. synchronous generators are the backbone of all our present-day electricity generation and transmission system. The system excitation of synchronous generator has not usually used permanent magnet excitation because of the requirements of assuring the powersystem stability. Also in the case of high power synchronous motors, the permanent magnet excitation is not recommended because of the requirements of power factor control irrespective of the mechanical load taken from the shaft. So, in these cases is highly recommended controlled excitation system. Static excitation is widely applied and shows considerable advantages in economics and in overall power-system stability.

There is two basic way of using static synchronous machine excitation [4]:

-Direct excitation of the machine field coils from a static power supply, d.c. electric energy have been transferred to the rotating rotor by means of a slip ring collector and brushes (Fig. 1).

-Brush-less generator system. The use of a rotating a.c. exciter generator with diodes to rectify its output to feed the main generator field, using a controlled static rectifier to supply the exciter generator field current (Fig. 2).

\section{Ring Colector}

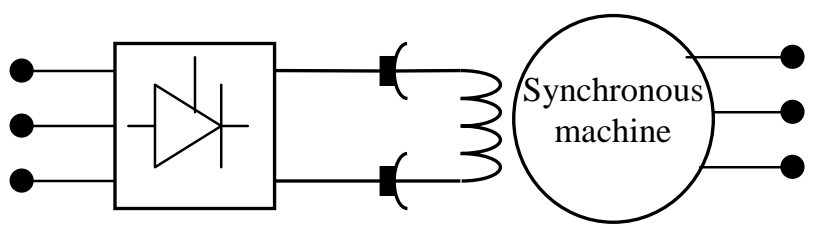

Fig. 2. Direct excitation system.

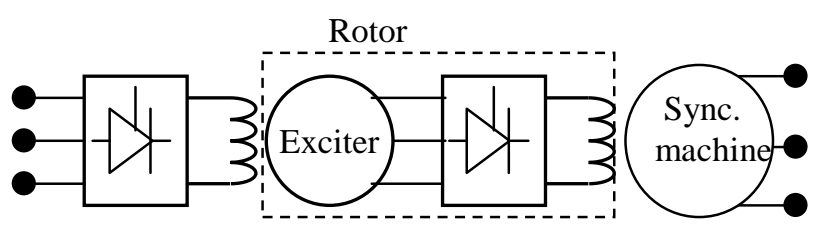

Fig. 3 Brush-less excitation system.

Response time for direct excitation system will be very fast and this can contribute significantly to the ability of the synchronous machine to cope with sudden load demands and system faults. But the slip ring-brushes collector don't work properly at high speed and for high power (about $2 \%$ from the power of main generator), and needs a permanent maintenance.

This slip ring-brushes system has a very high efficiency and low cost but has and some disadvantages as:

- Limited permissible speed;

-Low reliability;

- Sparkling effects, sensitivity against dust;

-Wear and tear and maintenance costs.

The brush-less excitation system has many attractions to the user from the maintenance point of view. But the increased overall time constant caused by the additional rotating machine may lead to a higher degree of field forcing been needed on the exciter alternator.

To avoid the maintenance problems of the first system and the high time constant of exciter coil of the later, we proposed a third type of static excitation system for synchronous electrical machines, contact-less excitation power supply (Fig. 1).

The contact-less excitation power supply for synchronous machines, proposed in this paper, consists of a rotating transformer RT with the primary side (winding and magnetic core) separated by the secondary side (winding and magnetic core) by a small air gap. Because of the presence of air-gap the frequency will be increased. The primary side will be fed from a high frequency inverter and will be standstill, fixed to the machine stator like brushes holder. The secondary side will be placed on the rotor on the same way as at brush-less excitation system, and it will feed the excitation coil by means of a rectifier, also placed on the rotor.

The excitation level can be controlled from the primary side, by changing the commutation frequency of primary inverter (series resonant inverter). But, also, this rotating rectifier can be fully commanded from outside, because signal and data transfer is also possible with the same rotating transformer RT with minimal additional hardware.

In such a way the response time will be small as in the case of direct excitation system, and the slip ring collector will be avoided as in the case of brush-less excitation system.

\section{Contact-less Excitation System (CES) - Design Procedure}

The goal of the design calculations is the determination of main geometrical dimensions of rotating transformer, which can be shown in figure 4 .

\section{A. Magnetic core design}

It is starting from the well-known Faraday's law:

$$
\left\{\begin{array}{l}
u_{2}=-N_{2} \cdot \frac{d \varphi}{d t}=-N_{2} \cdot \omega \cdot \hat{\Phi}_{2} \cdot \cos \omega t=\hat{U}_{2} \cdot \cos \omega t \\
U_{2}=\frac{\hat{U}_{2}}{\sqrt{2}}=\pi \cdot \sqrt{2} \cdot f \cdot N_{2} \cdot \hat{\Phi}_{2}
\end{array},\right.
$$

where: $\varphi_{2}=\hat{\Phi}_{2} \cdot \sin \omega, \Phi_{2}{ }^{\wedge}=S_{F e} * B_{F e}$ is the peakvalue of magnetic flux coupling the secondary winding, and $\omega=2 \pi f$ - is the angular frequency and $f$ is the frequency. 
The rated power will be:

$$
\begin{aligned}
& P_{2}=U_{2} \cdot I_{2}=\frac{\hat{U}_{2} \cdot \hat{I}_{2}}{2}= \\
& \pi \cdot f \cdot \hat{\Phi}_{2} \cdot\left(N_{2} \cdot \hat{I}_{2}\right)=\pi f \hat{\Phi}_{2} \hat{F}_{2}
\end{aligned}
$$

From this relation we can see that to increase the power the best way is to increase the frequency because the increasing of magnetic flux lead to large transversal section area of magnetic core and the increasing of magneto-motive force $\left(F_{2}=N 2 I 2\right)$ lead to increasing the copper mass. The increasing of the copper mass means the increasing of magnetic core window and hence and of the core mass.

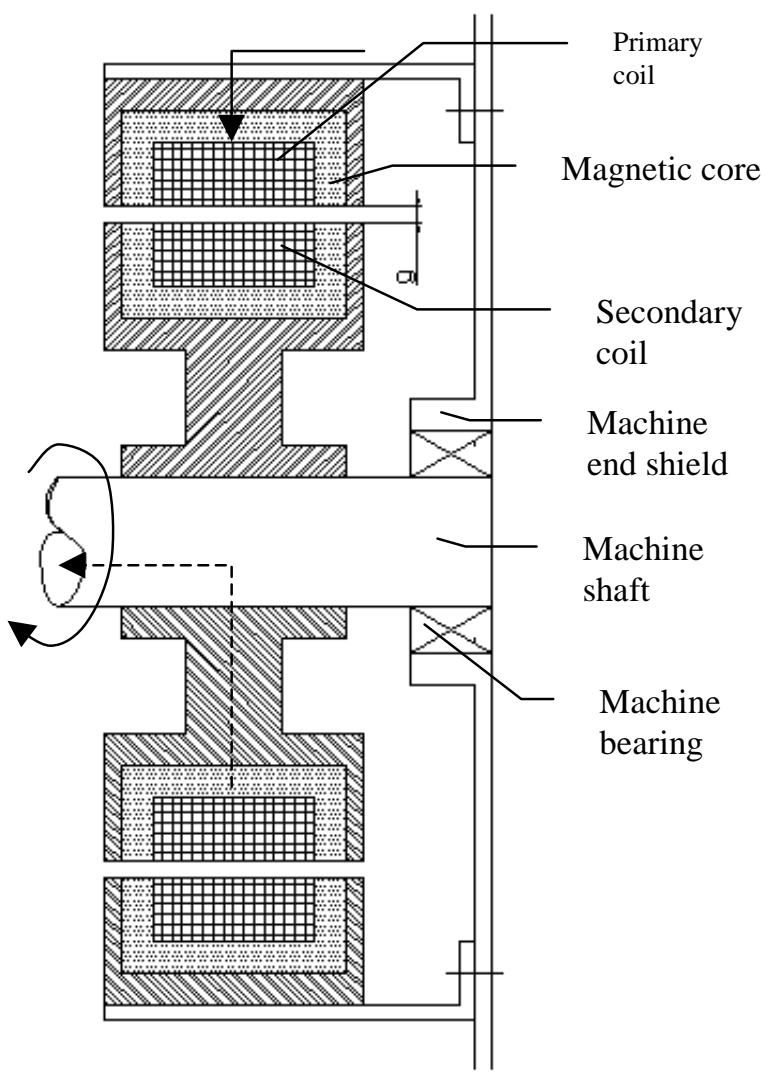

Fig. 4 CES construction.

Another problem is the presence of a very large air-gap of the magnetic core, about $g=5 \mathrm{~mm}$. This large air-gap requires a very large magnetizing current, according to the Ampere's law written below:

$$
\begin{aligned}
& F_{2 \mu}=\oint_{C} \vec{H} \cdot d \vec{l}=H_{F e} \cdot l_{F e}+H_{g} \cdot g= \\
& \frac{B_{F e} \cdot l_{F e}}{\mu_{r F e} \cdot \mu_{0}}+\frac{B_{g} \cdot 2 g}{\mu_{0}}=N_{2} \cdot i_{2 \mu}
\end{aligned},
$$

where: $N_{2}$ - is the number of turns of primary windings; $\mathrm{i}_{2 \mu}$ - is the transformer magnetizing current; $g$ - is the airgap length; $B_{g}-$ is the magnetic flux density in the airgap; $B_{F e}-$ is the magnetic flux density in the ferrite magnetic core; $l_{F e}$ - is the length of magnetic flux line in the ferrite core; $\mu_{0}-$ is the air magnetic permeability and $\mu_{r F e}-$ is the relative magnetic permeability of ferrite material.

Because of the high value of the $\mu_{r F e}(>1000)$ the first term is negligible. The secondary term, usually do not appear in a classical transformer, but now it has a high value. This value requires a high magnetizing current. In such a way we must to enlarge the dimensions of magnetizing coil.

If in a normal transformer, the magnetizing current is very small (about $2 \%$ from rated current), here, because of the presence of big air-gap, the magnetizing current can be estimated of the same value as the rated secondary current:

$$
I_{2 \mu}=I_{2} \cdot k_{m},
$$

where $k_{m}$ is the magnetizer coefficient.

From (2-4) it can be obtained:

$$
S_{F e}=\frac{P_{2} \mu_{0} k_{m}}{2 \pi f B_{F e} B_{g} g} .
$$

If we imposed the values for magnetizing coefficient $k_{m}$, frequency $f$, the air-gap length $g$, the magnetic flux density in the air-gap $B_{g}$ and in the core $B_{F e}$, we can calculate the cross-section area of the magnetic core $S_{F e}$. If it is imposed the inner diameter of magnetic core $D_{l}$, similar with a classical slip ring collector, we can calculate the minimum thickness of magnetic core in the worst place - the inner ring core (inner yoke):

$$
a=\frac{S_{F e}}{\pi D_{1}} .
$$

From the symmetry reasons this thickness will be adopted al around the core window.

The inner diameter of secondary coil will be:

$$
D_{2}=D_{1}+2 a \text {. }
$$

The overall secondary current has two components:

$$
i_{2 e}=i_{2}+i_{2 \mu},
$$

where $i_{2}$ is the load current and $i_{2 \mu}$ is the magnetizing current, but they are adding as vectors. Because the first current is almost active and the latter is pure inductive and the magnetizing current is of the comparable value as the load current we can assume that the overall current is:

$$
\begin{gathered}
\hat{I}_{2 e} \approx \sqrt{\hat{I}_{2}^{2}+\hat{I}_{2 \mu}^{2}}, \\
\hat{F}_{2 e}=N_{2} \hat{I}_{2 e}=2 \cdot S_{w 2} \cdot J_{a 2} \cdot k_{f 2},
\end{gathered}
$$

where $S_{w 2}$ is the primary window area; $J_{a}$ is the permissible current density and $k_{f l}$ is the filling factor of secondary window.

From here we can calculate the secondary window area and then all the geometrical dimensions of the core window.

The primary core window can be a little small than the primary because of the lack of magnetizing current, but from symmetry reasons we can take the primary side of the same shape and dimensions as the secondary side.

Now we will calculate the primary winding cross-section aria (from 10): 


$$
S_{w 2}=\frac{\hat{F}_{2 e}}{2 \cdot J_{a} \cdot k_{f 2}} .
$$

\section{B. Transformer Coils Design}

Now, we can design and the primary and secondary windings. From relation (1) we can write:

$$
N_{2}=\frac{\sqrt{2} \cdot U_{2}}{2 \cdot \pi \cdot f \cdot B_{F e} \cdot S_{F e}} .
$$

Admitting a coupling factor of $k_{c}$, the primary number of turns will be:

$$
N_{1}=N_{2} \cdot \frac{U_{1} \cdot k_{c}}{U_{2}} \text { turns. }
$$

The secondary magnetizing current will be:

$$
I_{2 \mu}=\frac{F_{\mu}}{N_{2}} .
$$

The secondary load current will be:

$$
I_{2}=\frac{P_{2}}{U_{2}} \text {. }
$$

It can be seen that the assumption (7) is true.

The effective secondary conductor section area is:

$$
S_{C u 2}=\frac{I_{2 e}}{J_{a}}=\frac{\sqrt{I_{2}^{2}+I_{2 \mu}^{2}}}{J_{a}} .
$$

The skin effect has not been taken in account because it is supposed that the primary winding is made of the litzwires. According with [6] for a frequency of $20 \mathrm{kHz}$ is recommended a litz wire of type $33 \mathrm{AWG}$ or more. It was chosen cable of type 38 AWG with $74 \mathrm{~mA}$ admissible current. Now we can calculate the number of strands in the litz-wire conductor of 38AWG type:

$$
N_{w 1}=\frac{I_{1 e}}{0.074} \text { strands. }
$$

The overall diameters of this kind of conductor single served is [6]:

$$
d_{w 1}=\sqrt{N_{w 1}} \cdot d_{w 1} \cdot 1.155
$$

\section{Electrical parameters}

The primary leakage inductance can be calculated by

$$
L_{s 1}=\frac{N_{1}^{2}}{R_{m s 1}}=\frac{N_{1}^{2} \cdot \mu_{0} \cdot \pi \cdot D_{1} \cdot g}{h}
$$

According with [6] the specific resistance for 38AWG is $0.65 \mathrm{ohm} /$ foot. For primary coil the overall resistance will be:

$$
R_{1}=\frac{0.65 \cdot l_{m 1} \cdot N_{1}}{N_{w 1}}
$$

On a similar way as the primary resistance, if $l_{2 \mathrm{~m}}$ is the average length of secondary coil conductor:

$$
R_{2}=\frac{0.65 \cdot l_{m 2} \cdot N_{2}}{N_{w 2}}
$$

The secondary leakage inductance can be calculated by:

$$
L_{s 2}=\frac{N_{2}^{2}}{R_{m s 2}}=\frac{N_{2}^{2} \cdot \mu_{0} \cdot \pi \cdot D_{2} \cdot g}{h}
$$

In order to calculate the magnetizing inductance, we will firstly calculate the main magnetic reluctance:

$$
R_{m}=R_{m F e}+R_{m g}=\frac{l_{F e}}{\mu_{r} \cdot \mu_{0} \cdot S_{F e}}+\frac{2 g}{\mu_{0} \cdot S_{g}},
$$

and then:

$$
L_{m}=\frac{N_{1} N_{2}}{R_{m}}=\frac{N_{2} L_{m}^{\prime}}{N_{1}}=\frac{N_{1} L_{m}^{\prime \prime}}{N_{2}} .
$$

These parameters can be more accurately calculated by a FEM analyses.

$$
\begin{aligned}
& L_{11}=L_{m}^{\prime}+L_{\sigma 1}, \\
& L_{22}=L^{\prime \prime}{ }_{m}+L_{\sigma 2},
\end{aligned}
$$

and from here we can calculate the coupling factor:

$$
k_{c}=\frac{L_{m}}{\sqrt{L_{11} L_{22}}} .
$$

Now we can build the equivalent scheme of a compensated rotating transformer:

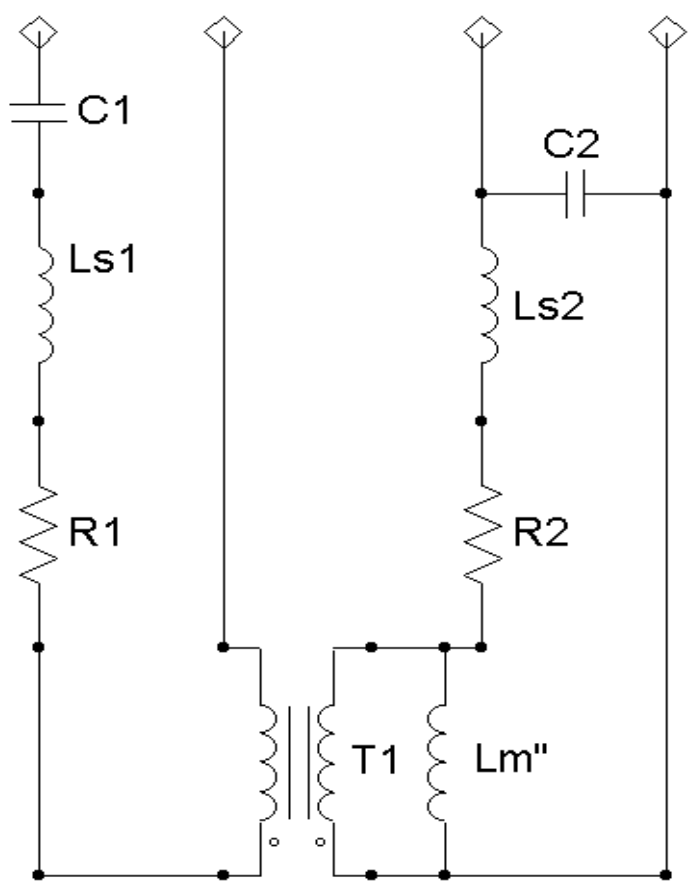

Fig. 5 The equivalent scheme of a rotating transformer with magnetizing inductance moved to the secondary side.

In the above scheme appear two compensating capacitors.

The series capacitor $\mathrm{C}_{1}$ has been introduced to 
compensate the leakage inductance of primary and secondary coil. It is possible to calculate this capacitor from the condition of running at resonance:

$$
C_{1}=\frac{1}{4 \cdot \pi^{2} \cdot f^{2} \cdot\left(L_{s 1}+L_{s 2}^{\prime}\right)}
$$

The parallel capacitor $\mathrm{C}_{2}$, mounted in the secondary side, compensates the magnetizing inductance and generate an inductive current equal with the magnetizing current, so the high value magnetizing current is locally generated and is avoided to take it from the primary inverter.

$$
C_{2}=\frac{1}{4 \cdot \pi^{2} \cdot f^{2} \cdot L_{m}^{\prime \prime}}
$$

\section{Application}

The design methodology presented before will be used to electromagnetic design of a contact-less excitation system (CES), for a small synchronous hydro-generator of $500 \mathrm{~kW}$ rated power.

\section{A. Design input data}

Taking in account that the needed excitation power of a synchronous electric motor is about $2 \%$ from the main machine power, the rated power of RTES can be estimated. A lot of initial data will be also imposed (Table I).

TABLE I. - Input Data

\begin{tabular}{|l|l|l|}
\hline Nr. & Parameter & Value \\
\hline 1 & Power $P[\mathrm{~kW}]$ & 10 \\
2 & Primary voltage $\mathrm{U}_{1}[\mathrm{~V}]$ & 220 \\
3 & Secondary voltage $\mathrm{U}_{2}[\mathrm{~V}]$ & 350 \\
\hline 4 & Frequency f[Hz $\}$ & 20000 \\
\hline 5 & Inner core diam. $\mathrm{D}_{1}[\mathrm{~mm}]$ & 110 \\
\hline 6 & Air-gap length $\mathrm{g}[\mathrm{mm}]$ & 5 \\
\hline 7 & Winding width $\mathrm{h}[\mathrm{mm}]$ & 20 \\
\hline 8 & Iron magn. flux dens. $\mathrm{B}_{\mathrm{Fe}}[\mathrm{T}]$ & 0.1 \\
\hline 9 & Air-gap magn. flux dens. $\mathrm{B}_{\mathrm{g}}[\mathrm{T}]$ & 0.02 \\
\hline 10 & Windows filling factor $\mathrm{k}_{\mathrm{u}}$ & 0.2 \\
\hline 11 & Coupling factor $\mathrm{k}_{\mathrm{c}}$ & 0.9 \\
\hline 12 & Magnetizer coefficient $\mathrm{k}_{\mathrm{m}}$ & 1 \\
\hline
\end{tabular}

\section{B. Design results}

Starting from this design data a Contactless Excitation System for a synchronous hydrogenerator of $500 \mathrm{kw}$ power, have been designed using the procedure presented before.

The imposed values are finally compared with the calculated ones, and if there are differences bigger than $10 \%$, the calculation must be resumed with new imposed values, as is usually recommended for nonlinear design problems.

The design results of this CES with input data presented in Table I are presented in Table II.
TABLE II. - Design Results

\begin{tabular}{|l|l|l|l|}
\hline Nr. & Parameter & Value & Unit \\
\hline 1 & Primary number of turns & 6 & turns \\
\hline 2 & Secondary number of turns & 10 & turns \\
\hline 3 & Primary conductor strands & 1000 & 38 AWG \\
\hline 4 & Secondary cond. strands & 1000 & 38 AWG \\
\hline 5 & Primary resistance & 0.007 & $\Omega$ \\
\hline 6 & Prim. leakage inductance & 2.7 & $\mu \mathrm{H}$ \\
\hline 7 & Second. resistance & 0.008 & \\
\hline 8 & Second. leak. Ind. & 7.5 & $\mu \mathrm{H}$ \\
\hline 9 & Magnetizing inductance & 37.5 & $\mu \mathrm{H}$ \\
\hline 10 & Series Capacitor & 12 & $\mu \mathrm{F}$ \\
\hline 11 & Parallel Capacitor & 0.9 & $\mu \mathrm{F}$ \\
\hline 12 & Outer diameter & 210 & $\mathrm{~mm}$ \\
\hline 13 & Maximum shaft diam. & 100 & $\mathrm{~mm}$ \\
\hline 14 & Air-gap diameter & 150 & $\mathrm{~mm}$ \\
\hline 15 & Magn. Core width & 10 & $\mathrm{~mm}$ \\
\hline 16 & Windows dimensions & $25 \mathrm{x} 10$ & $\mathrm{~mm}$ \\
\hline
\end{tabular}

\section{CPS - Operation Simulation}

An operation simulation using Electronics Workbench software has been performed. The simulation results are presented in figure 6 .

The output voltage dependence of frequency is presented in figure 7. It can be seen that the amplification in voltage is bigger than 1 (because of the resonance phenomena) in a large frequency range $(10-70 \mathrm{kHz})$.

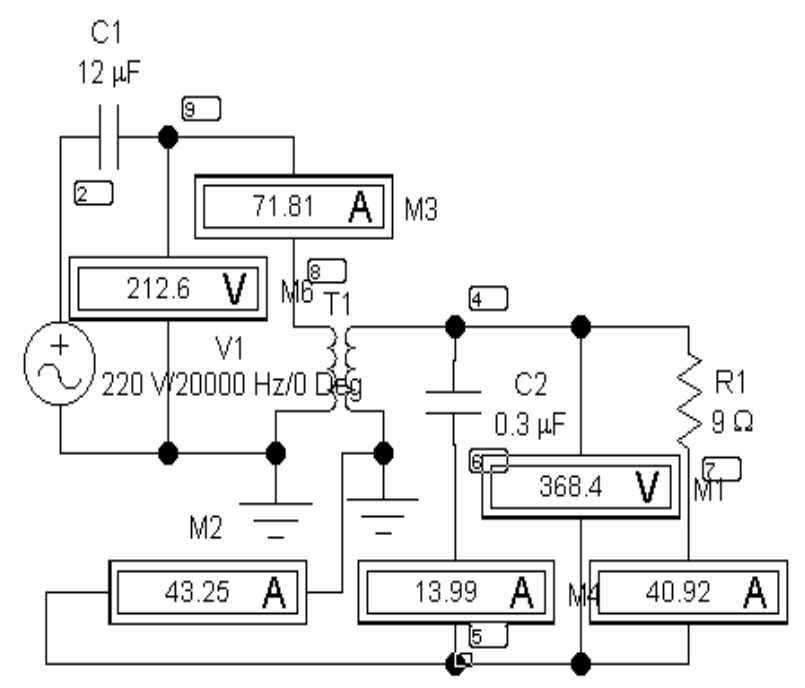

Fig. 6. Simulation results, output voltage depending on frequency.

From this picture (Fig. 7) we can note that the recommended frequency range is $18 . .30 \mathrm{kHz}$, where the output voltage remain relative constant.

It can be noticed that this characteristic is like a resonance characteristic, but the resonance peak is not so sharp, so it is not possible to use the changing the commutation frequency in order to regulate the load power. In this case the regulation of load power 
(excitation current), is recommended to be done by controlling the secondary side rectifier (Fig. 1). In such a sense can be used a supplementary pear of windings placed on the same ferrite core for transmitting of controlling signals (data transmission). These windings can use a different working frequency $(\mathrm{MHz})$, much different than power winding frequency.

In the following will be presented the simulation results for the case of frequency of $20 \mathrm{kHz}$.
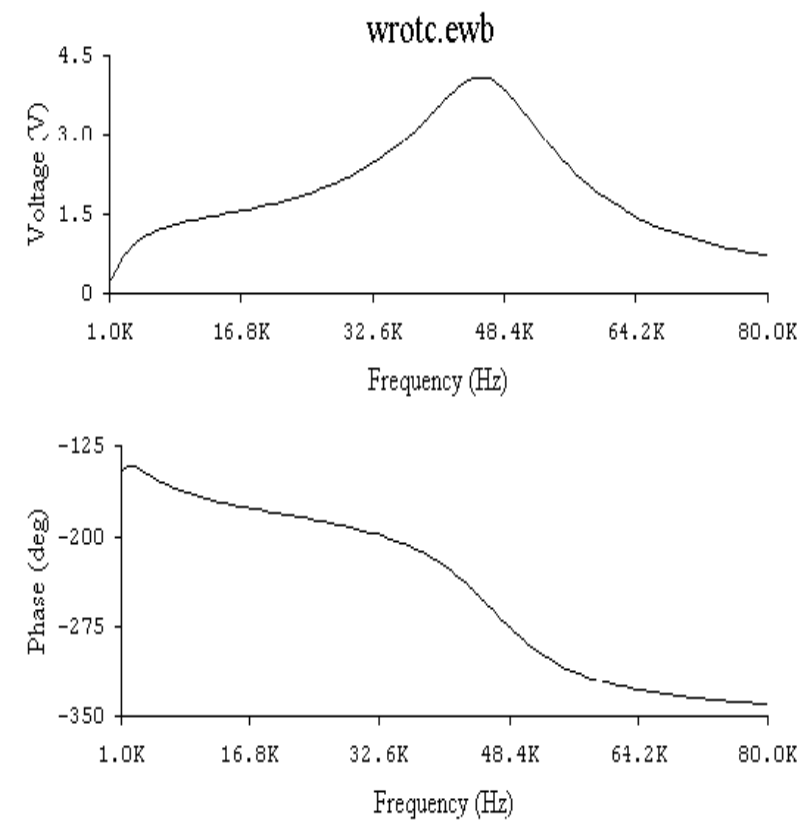

Fig. 7 The currents and voltages value of CES.

In the Table III are presented a lot of rated parameters in comparison with simulated parameters from figure 6 .

TABLE III Rated And Simulated Parameters

\begin{tabular}{|l|l|l|}
\hline Parameter. & Rated val. & Simul.val. \\
\hline Power [kW] & 10 & 15 \\
\hline Primary current [A] & 45.4 & 71.81 \\
\hline Secondary current [A] & 32 & 43.25 \\
\hline Load current & 28.57 & 40.92 \\
\hline Secondary voltage [V] & 350 & 368.4 \\
\hline
\end{tabular}

The secondary voltage is $368.4 \mathrm{~V}_{\mathrm{rms}}$, a little higher than design data $\left(350 \mathrm{~V}_{\mathrm{rms}}\right)$, and the currents have higher values than design data. This can be explained by the fact that the active power delivery is greater than rated power $(15 \mathrm{~kW}>10 \mathrm{~kW})$. All the simulated currents are in the same rapport with rated currents as the rapport of powers.

\section{Conclusions}

This paper presents a Contact-less excitation system for a synchronous machine (CES) - design procedure and operation simulation.
The design procedure is validated by operation simulation of a designed CES. The simulation parameters are very closed to design parameters.

The results are used as data design for the power converters for the contactless excitation system. This simulation allows to chose the configuration and the values for compensation capacitors of the whole electronic scheme of CES.

The contactless excitation system of synchronous machines has many advantages compared with classical excitation systems:

- No brushes or rings wear ensures that the CES system is virtually maintenance free.

- Speed: with slip ring collector speed is a limiting factor. With CPS speed of operation is unlimited.

-Safety: All components are fully enclosed and insulated. Hence the system is fully touch proof.

-Sensitive Environments: The fact, that no carbon dust, or other wear or sparks is generated, make CPS suitable for sensitive or hazardous environments.

- At the same rated power, the volume and mass of CES is of the same value like slip ring-brushes excitation system and about 3 times lower compared with brushless excitation systems.

- Allows the data transmission in both directions: from the rotor side to stator side and viceversa.

- Has a very low mechanical time constant because the rotating transformer mass is very low (high frequency).

\section{References}

1. Dae Hyun Koo, Do Hyun Kang and Ioan Vadan, Contactless Power Supply for High Speed Rotating Rotor, in Procc. Of KIEE Spring Annual Conference 2001, April 19-21 Jeju, Korea, pp. B20-B24.

2. Jurgen Meins, Contactless Power Supply for Transport Systems, Procc. Of MAGLEV'98, Damanashi, Japan, pp. 268273.

3. M. Jufer, and M. Perrottet, Contactless Energy and Information Transmission for Electric Drives, Procc. Of ELECTROMOTION'95, Cluj-Napoca, Romania, May 25-26, 1995, pp. 1-8.

4. D. Finney, The Power Thyristor and its Application, MacGraw-Hill, 1980, London, UK.

5. *** Soft Ferrite Catalog, CORE, PO_DONG SHIHUNG, Korea.

6. *** Litz-Wire Catalogue, WIRE TRONIC INC. http://www.wiretronic.com/.

7. Nathan Grossner, Transformers for Electronic Circuits, MacGraw-Hill Book Company, London, New York, Tokio, 1984.

8. I. Daescu, Parameters acquisition system for hydrogenerator rotor with rotating rectifying diodes, Energetica, vol. 51. No. 8, 2003, Bucharest, Romania, pp.366-369.

9. G. Florea, Musat E., Fast des-excitation method for hydrogenerators with rotating rectifying diodes, Energetica, vol. 51 . No. 8, 2003, Bucharest, Romania, pp.354-357. 